\section{POS1321 MENTAL HEALTH SCREENING IN PATIENTS WITH JUVENILE IDIOPATHIC ARTHRITIS}

S. Eulert ${ }^{1}$, M. Niewerth ${ }^{1}$, J. Hörstermann ${ }^{1}$, C. Sengler ${ }^{1}$, D. Windschall ${ }^{2}$, T. Kallinich ${ }^{3}$, J. Grulich-Henn ${ }^{4}$, F. Weller-Heinemann ${ }^{5}$, I. Foeldvari' ${ }^{6}$, S. Hansmann ${ }^{7}$, H. Baumeister ${ }^{8}$, R. Holl ${ }^{9}$, D. Staab ${ }^{3}$, K. Minden ${ }^{1,3}$. ${ }^{1}$ Deutsches Rheuma-Forschungszentrum, Programmbereich Epidemiologie, Berlin, Germany; ${ }^{2}$ St. Josef-Stift Sendenhorst, Abt. für Kinder- und Jugendrheumatologie, Sendenhorst, Germany; ${ }^{3}$ Charité - Universitatsmedizin Berlin, Klinik für Pädiatrie mit Schwerpunkt Pneumologie und Immunologie, Berlin, Germany; ${ }^{4}$ Universitätsklinikum Heidelberg, Zentrum für Kinderund Jugendmedizin, Heidelberg, Germany; ${ }^{5}$ Klinikum Bremen-Mitte, Prof.-Hess-Kinderklinik, Bremen, Germany; ${ }^{6} \mathrm{Hamburger}$ Zentrum für Kinder- und Jugendrheumatologie, Hamburger Zentrum für Kinder- und Jugendrheumatologie, Hamburg, Germany; ${ }^{7}$ Universitätsklinikum Tübingen, Klinik für Kinderheilkunde und Jugendmedizin, arcT, Tübingen, Germany; ${ }^{8}$ Universität Ulm, Institut für Psychologie und Pädagogik, Ulm, Germany; ${ }^{9}$ Universität UIm, ZIBMT, Institut für Epidemiologie und med. Biometrie, UIm, Germany

Background: Mental disorders often begin in the vulnerable phase of adolescence and young adulthood. Young people with chronic diseases are particularly at risk. Early recognition of mental health problems is necessary in order to be able to support those affected in a timely and adequate manner. By implementing a web-based generic screening tool for mental health in routine care, patients with juvenile idiopathic arthritis (JIA) and mental health conditions can be identified and provided with targeted treatment.

Objectives: To investigate the prevalence of mental health conditions in young people with JIA in routine rheumatology care.

Methods: Mental health screening is implemented as an add-on module to the National Paediatric Rheumatology Database (NPRD). The current data was gathered over a period of 24 months. Patients complete the screening tool which includes the Patient Health Questionnaire ${ }^{1}$ (PHQ-9, score 0-27) and the Generalized Anxiety Disorder scale ${ }^{2}$ (GAD-7, score 0-21) via a web-based questionnaire. The cut-off for critical values in PHQ-9 and GAD-7 were defined as values $\geq$ 10. Simultaneously, other data, such as sociodemographic data, disease activity (cJADAS10, score $0-30$ ), functional status (CHAQ, score $0-3$ ) were collected as well.

Results: The analysis included 245 patients $(75 \%$ female) with a mean age of 15.7 years and a mean disease duration of 8.8 years. $38.8 \%$ of the patients had oligoarthritis (18.0\% OA, persistent/20.8\% OA, extended) and $23.3 \%$ RF negative polyarthritis. At the time of documentation 49 patients $(30.6 \%)$ had an inactive disease (cJADAS10 $\leq 1)$ and $120(49.4 \%)$ no functional limitations $(\mathrm{CHAQ}=$ 0). In total, 53 patients $(21.6 \%$ ) had screening values in either GAD-7 or PHD-9 $\geq 10$. Patients with critical mental health screening values showed higher disease activity and more frequent functional limitations than inconspicuous patients (cJADAS10 (mean \pm SD): $9.3 \pm 6$ vs. $4.9 \pm 4.9$; CHAQ: $0.66 \pm 0.6$ vs. $0.21 \pm 0.42$ ). When compared to males, females were significantly more likely to report either depression or anxiety symptoms ( $11.7 \%$ vs. $24.9 \%, p=0.031)$.

$17.6 \%$ of all patients with valid items for these data reported to receive psychological support, meaning psychotherapeutic support (14.5\%) and/or drug therapy $(8.6 \%)$. Among those with a critical mental health screening score, $38.7 \%$ received psychological support (psychotherapeutic support (35.5\%) and/or drug therapy $(16.1 \%))$.

Conclusion: Every fifth young person with JIA reported mental health problems, however, not even every second of them stated to receive psychological support. The results show that screening for mental health problems during routine adolescent rheumatology care is necessary to provide appropriate and targeted support services to young people with a high burden of illness.

REFERENCES:

[1] Löwe B, Unützer J, Callahan CM, Perkins AJ, Kroenke K. Monitoring depression treatment outcomes with the patient health questionnaire-9. Med Care. 2004 Dec;42(12):1194-201.

[2] Spitzer RL, Kroenke K, Williams JB, Löwe B. A brief measure for assessing generalized anxiety disorder: the GAD-7. Arch Intern Med. 2006 May 22; 166(10):1092-7.

[3] The screening data were collected as part of COACH (Conditions in Adolescents: Implementation and Evaluation of Patient-centred Collaborative Healthcare), a project supported by the Federal Ministry of Education and Research (FKZ: 01GL1740F).

Disclosure of Interests: Sascha Eulert: None declared, Martina Niewerth: None declared, Jana Hörstermann: None declared, Claudia Sengler: None declared, Daniel Windschall: None declared, Tilmann Kallinich: None declared, Jürgen Grulich-Henn: None declared, Frank Weller-Heinemann Consultant of: Pfizer, Abbvie, Sobi, Roche, Novartis, Ivan Foeldvari Consultant of: Gilead, Novartis, Pfizer, Hexal, BMS, Sanofi, MEDAC, Sandra Hansmann: None declared, Harald Baumeister: None declared, Reinhard Holl: None declared, Doris Staab: None declared, Kirsten Minden: None declared DOI: 10.1136/annrheumdis-2021-eular.3249

\section{POS1322 \\ A DATA SCIENCE EVALUATION OF THE JUVENILE ARTHRITIS MULTIDIMENSIONAL ASSESSMENT REPORT (JAMAR) QUESTIONNAIRE FOR IMPROVING MANAGEMENT OF JIA PATIENTS}

H. Quesada-Masachs ${ }^{1}$, M. Faloutsos ${ }^{1}$, M. López- Corbetó ${ }^{2}$,

S. Ghose ${ }^{3}$, S. Marsal ${ }^{4}$, E. Quesada-Masachs ${ }^{2} .{ }^{1}$ University of California Riverside, Computer Science \& Engineering, Riverside, United States of America; ${ }^{2}$ Hospital Universitari Vall d'Hebron, Rheumatology, Pediatric Rheumatology Unit, Barcelona, Spain; ${ }^{3}$ University of California Berkeley, University of California Berkeley, Berkeley, United States of America; ${ }^{4}$ Hospital Universitari Vall d'Hebron, Rheumatology, Barcelona, Spain

Background: The Juvenile Arthritis Multidimensional Assessment Report (JAMAR) is a questionnaire developed to comprehensively assess Juvenile Idiopathic Arthritis (JIA) patients. Despite being translated into 54 languages, there is still limited literature about it. The length of the questionnaire could have been influencing its clinical practicality. Objectives: The purpose of this study is to answer the following questions: a) "Which are the most informative questions?";

b) "How well do the collected data correlate with other clinical variables?"; c) "Are there discrepancies between the perceptions of patients and parents?"; Methods: We included 71 children with JIA according to ILAR criteria, all of them receiving treatment and we followed them up for a year. JAMAR questionnaires were answered by both children and parents at baseline, 6 and 12 months. Also, a thorough clinical examination was performed in every visit: all the joints were clinically assessed for swelling, tenderness, and limited range of motion, and Juvenile Arthritis Disease Activity Score (JADAS), disease activity state, parents and patients assessment through Visual Analogue Scale (VAS), physician's VAS, Erythrocyte Sedimentation Rate (ESR) and C-reactive protein (CRP) were recorded. We applied state of the art machine learning methods in order to find the most relevant questions in JAMAR. Additionally, we utilized tensor decomposition to identify relevant patient clusters. Furthermore, we correlated these critical questions with clinical and biological parameters recorded. We have compared the discordance rate between patients vs parents responses in 5 of JAMAR parameters as previously reported [1]. We explored the relation between discordance and demographic and clinical variables.

Results: A total of 374 JAMAR questionnaires are analyzed with our Machine Learning algorithms. First, we identify a small group of questions as the most relevant for patients and parents. The identified questions exhibit better correlations with the JADAS scores than the non-relevant ones. Second, $96 \%$ of the pairs (child-parent) are discordant for at least one item, but the differences are small and VAS well being is the only score with a statistically significant difference $(P<0.0001)$. We observe a higher rate of activity in the patients exhibiting discordant evaluations with their parents. In addition, the observation patient-parent agreement in Juvenile Arthritis Functionality Scale (JAFS) is better than Pediatric Rheumatology Quality of Life Scale (PRQL).

Conclusion: In this study, we revisited the JAMAR questionnaire by applying modern data mining techniques in a longitudinal dataset. Our results suggest that a small number of questions in the JAMAR questionnaire provide significant information and correlate well with the JADAS scores. We argue that this reduced set of questions could make the data collection easier by trading off the number of questions for frequency and ease of self-reported data collection.

\section{REFERENCES:}

[1] Vanoni $F$, et al. The difference of disease perception by juvenile idiopathic arthritis patients and their parents: analysis of the JAMAR questionnaire. Pediatr Rheumatol Online J. 2016;14(1):2.

Disclosure of Interests: None declared

DOI: 10.1136/annrheumdis-2021-eular.3319

\section{POS1323 SACROILIAC JOINT MRI ABNORMALITIES IN JUVENILE SPONDYLOARTHRITIS: AN UPDATE OF DEFINITIONS AND SCORING OF THE OMERACT JUVENILE IDIOPATHIC ARTHRITIS MRI SCORE}

T. M. Otobo ${ }^{1,2,3}$, N. Herregods ${ }^{4}$, J. L. Jaremko ${ }^{5}$, R. G. Lambert ${ }^{5}$, I. SudołSzopińska $^{6}$, A. B. Meyers ${ }^{7}$, E. Kirkhus ${ }^{8}$, P. Weiss ${ }^{9}$, S. M. Tse ${ }^{10}$, S. Appenzeller ${ }^{11}$, P. G. Conaghan ${ }^{12}$, D. G. Rumsey ${ }^{13}$, J. Stimec ${ }^{1}$, L. Jans ${ }^{4}$, M. Van Rossum ${ }^{14}$, N. Tzaribachev ${ }^{15}$, J. Carrino ${ }^{16}$, O. Papakonstantinou ${ }^{17}$, M. Tolend ${ }^{1,2,3}$, R. Moineddin ${ }^{18}$, N. Haroon ${ }^{19,20}$, W. P. Maksymowych ${ }^{21}$, A. S. Doria ${ }^{1,3,22}$ on behalf of Outcome Measure in Rheumatology Juvenile Idiopathic Arthritis MRI - Sacroiliac Joint Special Interest Group (OMERACT JAMRI-SIJ). ${ }^{1}$ Hospital for Sick Children, Diagnostic Imaging, Toronto, Canada; ${ }^{2}$ University of Toronto, Institute of Medical Science, Toronto, Canada; ${ }^{3}$ Hospital for Sick Children Research Institute, Translational Medicine, Toronto, Canada; ${ }^{4}$ Prinses Elisabeth Children's Hospital / Ghent University Hospital, Radiology and Nuclear Medicine, Ghent, Belgium; ${ }^{5}$ University of Alberta, Radiology and Diagnostic Imaging, Edmonton, Canada; ${ }^{6}$ National Institute of Geriatrics, Rheumatology and Rehabilitation, Radiology, Warsaw, Poland; ${ }^{7}$ Cincinnati Children's Hospital Medical Center, Radiology, Cincinnati, United States of America; ${ }^{8}$ Oslo University Hospital, Radiology, Oslo, Norway; ${ }^{9}$ Children's Hospital of Philadelphia and 